\title{
Ultrasound Image Denoising via Dictionary Learning and Total Variation Regularization
}

\author{
Shuai $\mathrm{LI}^{1, \mathrm{a}}$, Ximei ZHAO ${ }^{2, \mathrm{~b}}$ \\ ${ }^{1}$ College of Information Engineering, Qingdao University, Qingdao, 266071, China \\ ${ }^{2}$ College of Information Engineering, Qingdao University, Qingdao, 266071, China

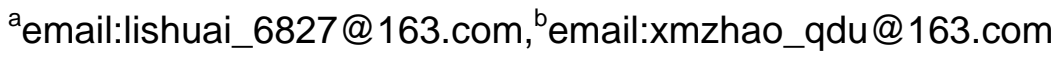
Keywords: Image Denoising; Multiplicative Noise; Dictionary Learning; TV Regularization;
Ultrasound Image

\begin{abstract}
The restoration of images corrupted by speckle noise is a key issue in medical images. In this paper, a novel sparse model is presented for speckle reduction in ultrasound (US) images. This model contains three terms: a patch-based sparse representation prior over dictionary learning, a pixel-based total variation (TV) regularization term, and a data-fidelity term capturing the statistics of Rayleigh noise. The split Bregman algorithm for the proposed model is presented to solve the optimization problem. Experimental results on real US images validate that the proposed method is able to keep accurately edges and preserve meaningful structural details of the images.
\end{abstract}

\section{Introduction}

Ultrasound (US) images are widely used in medical diagnosis because of its noninvasive. However, the diagnosis quality is often low since US images suffer from strong speckle noise. Thus, reducing speckle noise while preserving anatomic information is necessary to improve the quality and to enhance the diagnostic potential of US image.

Several methods have been proposed for the speckle reduction of US images. The Speckle Reducing Anisotropic Diffusion (SRAD) method [1] was proposed by casting a noise-dependent instantaneous coefficient of variation into diffusion model. Soon afterwards, methods with total variation (TV) minimization scheme have been applied to US imaging [2]. They used TV norm as the regularization term. Rayleigh distribution is used as modeling the distribution of noise in US imaging [3][4]. TV-based methods are iterative and provide smooth images while well preserving edges. Unfortunately, meaningful structural information is also removed during iterations. Buades et al. [5] proposed a patch-based nonlocal (NL) restoration method. This so-called NL-means filter replaces the local comparison of pixels with the nonlocal comparison of patches. In [6], Li et al. proposed a nonlocal TV model (NLTV) for multiplicative noise with Rayleigh distribution removal. They used nonlocal TV norm as the regularization term. The nonlocal-based methods lead to competitive results. However, the main drawback is its high computational cost.

Recently, it has been demonstrated that sparse representation of images is efficient approach for image recovery [7][8][9]. The basic principle of this method is that image patches have a nature of sparse representation. More precisely, each image patch can be sparsely represented as a linear combination of atoms taken from a fixed dictionary. Deka [9] used the dictionary learning method to reduce speckle noise which provides good results. The speckle noise removal scheme using sparse and redundant representations over learned dictionaries provides competitive results.

Accordingly, in this paper we combine the sparse model and TV regularization term to smooth ultrasound images. In what following, we will assume that the noise of ultrasound images follows Rayleigh distribution. Using maximum a posteriori (MAP) principle, we deduce the data-fidelity term capturing the statistic of Rayleigh noise. 


\section{Proposed model and its split Bregman algorithm}

In this paper, we have decided to choose the following general speckle model

$$
f=u^{*} n
$$

where $f$ is the degraded image, $u$ is the ideal image to recover and $n$ denotes multiplicative noise, which follows the Rayleigh distribution with mean one. The probability density function of $n$ is

$$
p d f(n)=\frac{n}{\sigma^{2}} \exp \left(-\frac{n^{2}}{2 \sigma^{2}}\right), n \geq 0
$$

where $\sigma$ is a parameter indicating the intensity of the noise.

Our goal is to estimate the ideal image $u$ from the observed image $f$. A classical statistical approach is the MAP estimator

$$
\hat{u}=\underset{u}{\arg \max } P(u \mid f)
$$

According to Bayes formula, the a posteriori density is $p(u \mid f) \propto p(f \mid u) p(u)$, so we deduce the data-fidelity term (4) capturing the statistic of Rayleigh distribution.

$$
\int_{\Omega}\left(\frac{1}{2}\left(\frac{f}{u}\right)^{2}+\log u\right) d x d y
$$

Images encountered in real applications often present lots of repeated patterns, in particular edges and txtures. This is probably why methods using sparse representation have provided promising results. In the proposed model, we use the sparse representation prior as a regularization term, and the data-fidelity term (4) to smooth ultrasound images. Moreover, TV-based methods can not only remove noise in flat region effectively, but also can reduce the artifacts in smooth regions caused by patch-based prior of dictionary learning in denoising tasks. Therefore, we add a TV regularization term. In conclusion, the discrete sparse model proposed for ultrasound image denoising can be written by

$$
\min _{\alpha, u}<1, \frac{1}{2}\left(\frac{f}{u}\right)^{2}+\log u>+\lambda\|\nabla u\|_{1}+\tau \sum_{i j}\left\|R_{i j} u-D \alpha_{i j}\right\|_{2}^{2}+\tau \sum_{i j} \mu_{i j}\left\|\alpha_{i j}\right\|_{0}
$$

where $\lambda, \tau$ are positive parameters that balance different terms. \|\|$_{1}$ is $l_{1}$-norm that sum the absolute values of $\nabla u$.

In model (5), $R_{i j} \in \square^{n \times N}$ is a $n \times N$ matrix which extracts a $\sqrt{n} \times \sqrt{n}$ patch from $u$ located in $(i, j)$ (thus, $R_{i j} u \in \square^{n}$ ). The first term is the data-fidelity term, the discrete version of (4), which captures the statistics of Rayleigh noise. The second term is the TV regularization term. The third part demands that each patch $R_{i j} u$ of the recovered image $u$ can be represented by the dictionary $D \in \square^{N \times K}$ with sparse coefficients $\alpha_{i j} \in \square^{K}$ with a bounded error. The fourth term is a sparsity constraint, which demands that the number of coefficients required to represent any patch is small. Note that the values $\mu_{i j}$ are hidden patch-specific weights that are determined by the optimization procedure. Clearly, this model mixes two priors: the TV regularization prior, which helps reduce the artifacts and keep sharp edges, and the sparse representation prior, which contributes to recover textures and preserve more structural details.

Split Bregman algorithm [10][11] has provided good results in splitting the seemingly unsolved variable optimization problem into several easy subproblems. Here, we introduce auxiliary variables $d_{1}=\nabla u, d_{2}=u$ to perform our algorithm. Dictionary learning methods cannot play a good effect when it is directly applied to multiplicative noise removal. So, the special constraint $d_{2}=u$ is added to solve this problem. Using the variable splitting method presented above, the minimization problem (5) becomes 


$$
\min _{\alpha, D, u, d_{1}, d_{2}}<1, \frac{1}{2}\left(\frac{f}{d_{2}}\right)^{2}+\log d_{2}>+\lambda\left\|d_{1}\right\|_{1}+\frac{1}{2 \rho}\left\|b_{1}+\nabla u-d_{1}\right\|_{2}^{2}+\tau \sum_{i j}\left\|R_{i j} d_{2}-D \alpha_{i j}\right\|_{2}^{2}+\frac{1}{2 \gamma}\left\|b_{2}+u-d_{2}\right\|_{2}^{2}+\tau \sum \sum_{i j} \mu_{i j}\left\|\alpha_{i j}\right\|_{0}
$$

where $\rho, \gamma$ are the penalty parameters.

An effective solution of split Bregman iteration of (6) is given below:

$$
\left\{\begin{array}{l}
u^{k+1}=\underset{u}{\arg \min } \frac{1}{2 \rho}\left\|b^{k}+\nabla u-d_{1}{ }^{k}\right\|_{2}^{2}+\frac{1}{2 \gamma}\left\|b_{2}{ }^{k}+u-d_{2}{ }^{k}\right\|_{2}^{2} \\
d_{1}^{k+1}=\underset{d_{1}}{\arg \min } \lambda\left\|d_{1}\right\|_{1}+\frac{1}{2 \rho}\left\|b_{1}+\nabla u-d_{1}\right\|_{2}^{2} \\
\alpha_{i j}=\underset{\alpha_{i j}}{\arg \min } \tau \sum_{i j}\left\|R_{i j} d_{2}-D \alpha_{i j}\right\|_{2}^{2}+\tau \sum_{i j} \mu_{i j}\left\|\alpha_{i j}\right\|_{0} \\
d_{2}{ }^{k+1}=\underset{d_{2}}{\arg \min }<1, \frac{1}{2}\left(\frac{f}{d_{2}}\right)^{2}+\log d_{2}>+\tau \sum_{i j}\left\|R_{i j} d_{2}-D \alpha_{i j}\right\|_{2}^{2}+\frac{1}{2 \gamma}\left\|b_{2}{ }^{k}+u^{k+1}-d_{2}\right\|_{2}^{2} \\
b_{1}{ }^{k+1}=b_{1}{ }^{k}+\nabla u^{k+1}-d_{1}{ }^{k+1} \\
b_{2}{ }^{k+1}=b_{2}{ }^{k}+\nabla u^{k+1}-d_{2}{ }^{k+1}
\end{array}\right.
$$

The equations about $u$ and $d_{2}$ in (7) are least squares problems. The equation about $\alpha_{i j}$ is the sparse coding stage in the K-SVD algorithm [7]. To solve it, we use OMP method [12] as suggested in the K-SVD algorithm. $b_{1}$ and $b_{2}$ are Bregman iteration parameters. The equation about $d_{1}$ is an $l_{1}$-regularized least squares problem. It can be solved by the generalized shrinkage formula, which is defined by $\operatorname{shrink}(a, b)=\max (|a|-b, 0) \frac{a}{|a|}$.Thus, the solutions of $u, d_{1}$ and $d_{2}$ in (7) are given as following:

$$
\begin{aligned}
& u^{k+1}=\frac{1}{(1 / \gamma+4 / \rho)} \cdot\left(\frac{1}{\gamma}\left(d_{2}{ }^{k}-b_{2}{ }^{k}\right)+\frac{1}{\rho}\left(u_{N}{ }^{k+1}+u_{S}{ }^{k+1}+u_{E}{ }^{k+1}+u_{W}{ }^{k+1}+\nabla \cdot b_{1}{ }^{k}-\nabla \cdot d_{1}{ }^{k}\right)\right) \\
& d_{1}^{k+1}=\operatorname{shrink}\left(\nabla u^{k+1}+b_{1}^{k}, \lambda \rho\right)=\max \left(\left|\nabla u^{k+1}+b_{1}^{k}\right|-\lambda \rho, 0\right) \frac{\nabla u^{k+1}+b_{1}^{k}}{\left|\nabla u^{k+1}+b_{1}^{k}\right|_{\varepsilon}} \\
& d_{2}{ }^{k+1}=\left(2 \tau \sum_{i j} R_{i j}{ }^{T} R_{i j}+\frac{1}{\gamma}\right)^{-1}\left(2 \tau \sum_{i j} R_{i j}{ }^{T} D \alpha_{i j}+\frac{1}{\gamma}\left(b_{2}{ }^{k}+u^{k+1}\right)+\frac{f^{2}}{\left(d_{2}{ }^{k}\right)^{3}}-\frac{1}{d_{2}{ }^{k}}\right)
\end{aligned}
$$

where $\varepsilon>0$ (in (9)) is a small disturbance to prevent the denominator to be zero.

Table 1 Overall algorithm

\section{Overall algorithm}

Initialization: Set $u^{0}=f, d_{1}^{0}=\nabla f, d_{2}^{0}=f, D=D C T$, and $b_{1}^{0}=b_{2}^{0}=0$.

for $i t e r_{0}=1$ to $N_{0}$ do

for iter $_{I}=1$ to $N_{I}$ do

update $u^{k+1}$ using (8);

update $d_{1}^{k+1}$ using (9);

update $\left\{\alpha_{i j}\right\}$ using OMP;

update $d_{2}{ }^{k+1}$ using (10);

update the Bregman iteration parameter $b_{1}^{k+1}, b_{2}^{k+1}$ using (7);

end

iter $_{I}=i t e r_{I}+1$;

update the dictionary $D$;

$\rho=\rho \cdot m_{1} ;$

$\gamma=\gamma \cdot m_{2} ;$ 
As we saw above, model (5) is equivalent to model (6) when the parameters $\rho, \gamma$ tend to ${ }^{+\infty}$. However, when we use too large values directly in the beginning, numerical problems occur in the optimal process. Hence, we begin with small values and gradually increase them in the iterative process. Moreover, the choice of $D$ has an important impact on the performance of the algorithm. In [7], it is shown that learning the dictionary from the noise image leads to better performance in most cases. Here, the dictionary $D$ is updated at the last step. We use the estimated image ${ }^{u}$ to learn $D$ in order to capture the distinctive features of the processed image. For saving the computational time, we just update the $D$ in the outer loop. Our whole procedure is summarized in Table 1.

Note that in Table 1, $N_{O}$ and $N_{I}$ represent the maximum number of iterations for the outer and inner loops. $m_{1}$ and $m_{2}$ denote that the growth rates of the parameters.

\section{Numerical experiments}

In this section, to validate the effectiveness of our model, we compare the proposed method to different ultrasound image denoising methods SARD [1], VO [13] and NLT [6]. VO is a model of image decomposition and the author also use it for US image denoising.

First, we compare different methods with experiments on synthetic image Barbara $(256 \times 256)$, with multiplicative Rayleigh noise of different levels $\sigma=0.2$ and $\sigma=0.4$. To assess denoising performance, the PSNR and MSSIM values [14] were computed between the observed image and its denoised version.

$$
P S N R=10 \log _{10} \frac{255^{2}}{\frac{1}{M \times N}\|\hat{u}-u\|^{2}}, M S S I M=\frac{1}{N} \sum_{i=1}^{N}[l(\hat{u}, u)]^{\alpha} \cdot[c(\hat{u}, u)]^{\beta} \cdot[s(\hat{u}, u)]^{\gamma}
$$

where $\hat{u}$ and $u$ denote the recovered image and the true original image respectively. $\mathrm{N}$ is the number of blocks of the processed image. $l(\cdot), c(\cdot)$ and $s(\cdot)$ are luminance, contrast, structure comparison functions respectively. $\alpha>0, \beta>0$ and $\gamma>0$ are weighted parameters that used to regulate the relative importance of the three components. The value is closer to 1 , the higher of fidelity between images that compared.

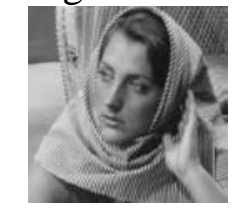

Original

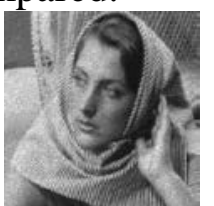

Noise

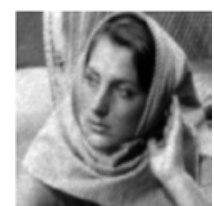

SRAD

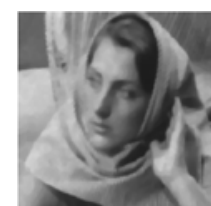

$\mathrm{VO}$

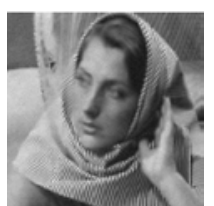

NLTV

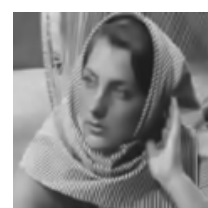

Proposed

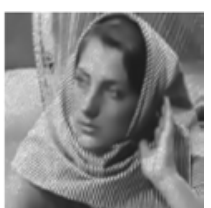

Without TV

Fig.1 Denoising results obtained with different methods applied to Brabara ( $\sigma=0.4)$.

Table 2 PSNR and MSSIM values obtained with several methods to Barbara

\begin{tabular}{lcc}
\hline \multirow{2}{*}{ Methods } & \multicolumn{2}{c}{ PSNR(dB) $\mid$ MSSIM } \\
\cline { 2 - 3 } & \multicolumn{1}{c}{$\sigma=0.2$} & $\sigma=0.4$ \\
\hline VRAD & $24.07 \mid 0.6673$ & $23.92 \mid 0.6532$ \\
NLTV & $24.93 \mid 0.6992$ & $24.90 \mid 0.6963$ \\
Proposed & $28.92 \mid 0.8216$ & $28.87 \mid 0.8188$ \\
Proposed-without TV & $\mathbf{3 1 . 7 4} \mid \mathbf{0 . 8 8 0 1}$ & $\mathbf{3 0 . 3 6} \mid \mathbf{0 . 8 6 4 7}$ \\
\hline
\end{tabular}

Fig. 1 show the denoising results obtained with different methods applied to the synthetic image Barbara with multiplicative Rayleigh noise $\sigma=0.4$. For each method and for each noise level, the PSNR and MSSIM values are given in Table 2. In this experiment, the parameters were adjusted to get the best PSNR values. For all levels of noise, the proposed method obtained the best PSNR and MSSIM values. As can be seen from Fig.1, the edges in the results using SRAD and VO model is blurred. Moreover, the structure information is also lost while removing noise. Although the result 
using the NLTV method maintains most structure information, it keeps too much noise to some extent. Visually, the proposed method satisfyingly removes noise while preserving edges and keeping meaningful structure information. In addition, we show the restored effect when TV term is not used in the proposed model ( $\lambda=0$ in model (5)). Obviously, the recovered result by proposed method has fewer image artifacts than that obtained by the model without TV term. Hence, the TV term is an important regularization term to reduce artifacts. Moreover, Table 2 shows that the proposed model achieves the best PSNR and MSSIM values.

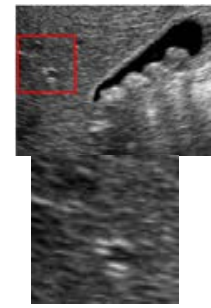

Original

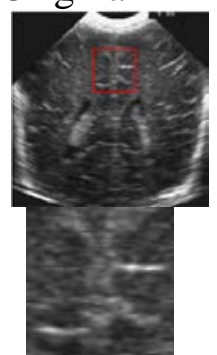

Original

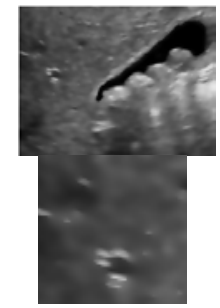

SRAD

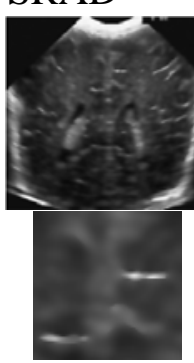

SRAD

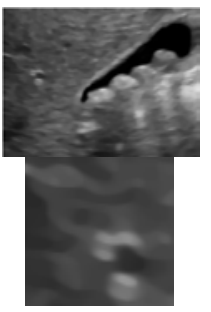

$\mathrm{VO}$

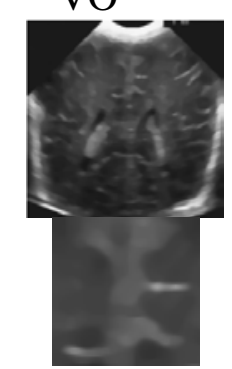

$\mathrm{VO}$

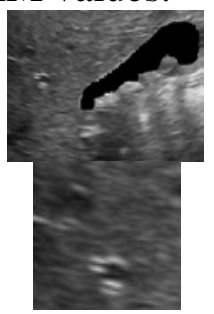

NLTV

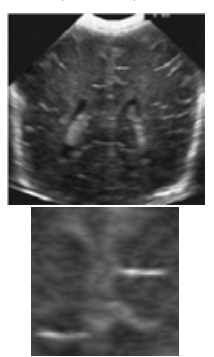

NLTV

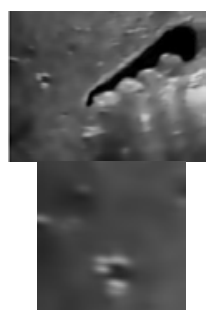

Proposed

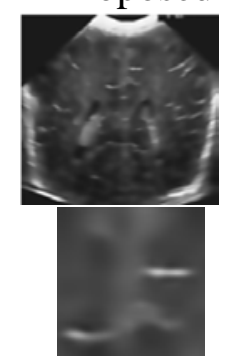

Proposed

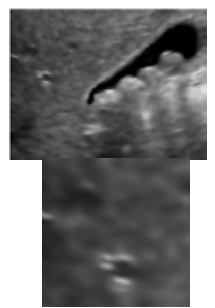

Without TV

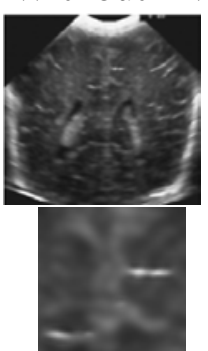

Without TV

Fig.2 Denoising Results obtained with different methods on real US images.

We also propose a visual comparison of our method and the other methods on real ultrasound images. The parameters for SRAD, VO and NLTV are those given respectively in [1], [13] and [6]. The parameters of the proposed model are set as follows: $\tau=50, N_{O}=10, N_{I}=60$, and the initialization for $\gamma$ and $\rho$ are 0.1 and 100. Growth rates are $m_{1}=1.5$ and $m_{2}=2$. Other parameters are chosen empirically in order to achieve good performance.

Fig.2 shows the experimental results applied to different ultrasound images with different methods. In order to compare the capability of preserving features effectively, the visual effects of partial-enlarged are also presented. Visually, the proposed model efficiently removes speckle noise while keeping the edges and preserving more structures. The visual results produced by our method are competitive compared to SRAD, VO and NLTV.

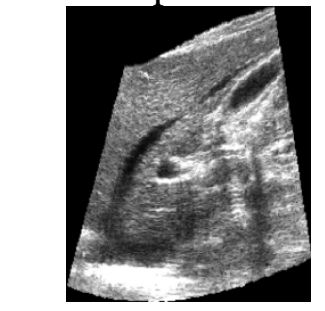

Original Liver-1

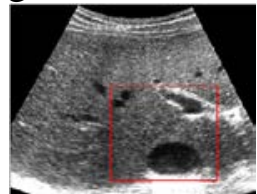

Original Liver-2

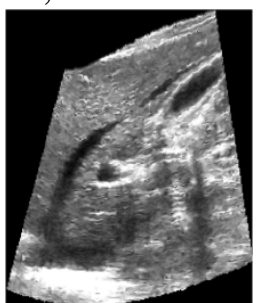

NLTV

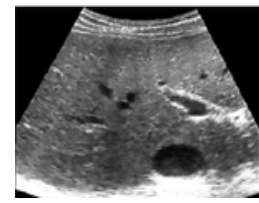

NLTV

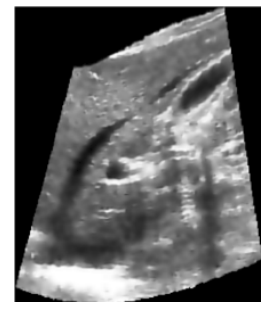

Proposed

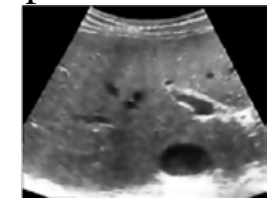

Proposed

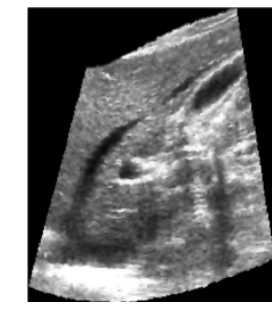

Proposed-without TV

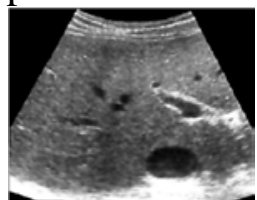

Proposed-without TV

Fig.3 Denoising results obtained with NLTV and Proposed model on Liver images. 


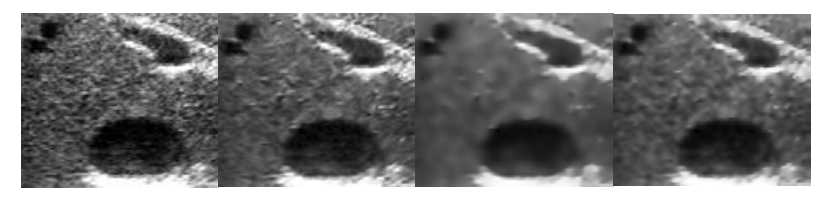

Original

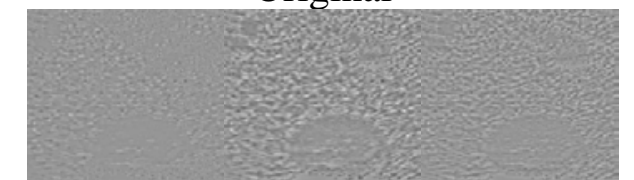

\section{NLTV Proposed Proposed-without TV}

Fig.4 Denoising results and the removed noise component with different methods.

In Fig.3, we propose to a visual comparison of the NLTV and the proposed method on real Liver images. It can be seen that our model remove speckle noise more effectively than NLTV. Fig.4 shows the partial-enlarged views of Liver- 2 in Fig. 3 and the removed noise component. The edge preservation of the proposed model is visible on the removed noise component with the least image structure details removed. In addition, the difference in dark areas and gray areas shows the smoothing adaptation according to the signal intensity. Clearly, the noise in brighter areas is drastically removed.

In all our experiments, the denoising process includes a sparse coding stage of each patch whose size is $8 \times 8$ from the noisy image. The dictionaries used of size $16 \times 256$ are learned from the degraded images directly. Then, it is interesting to see that the dictionary learned from Liver images shown in Fig. 5 contains some distinctive characteristics of the original images.

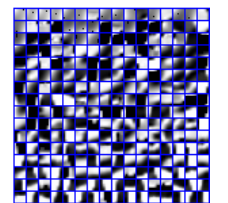

Liver-1

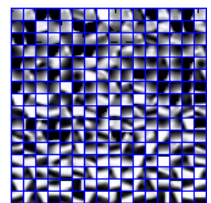

Liver-2

Fig.5 Dictionaries trained from real Liver images.

\section{Conclusion}

In this paper, a new sparse model dealing with ultrasound speckle noise was presented. Using MAP, we deduced the data-fidelity term capturing the statistics of Rayleigh noise. As for regularization terms, we combined sparse representation prior and TV regularization term to smooth ultrasound images. Experimental results show that the proposed model efficiently removes speckle noise while enhancing the edges and preserving meaningful image structures well.

\section{Acknowledgements}

This work was supported by National Natural Science Foundation of China under Grant 61303079.

\section{References}

[1] Yongjian Yu, Scott T. Acton. Speckle reducing anisotropic diffusion [J]. IEEE Transactions on Image Processing, 200211 (11) 1260-1270.

[2] Hyenkyun Woo, Sangwoon Yun, Myungjoo Kang. Speckle reduction variational model with total variation regularization [J]. Experimental Mathematics, 2011 161-163.

[3] TC Aysal, KE Barner. Rayleigh-maximum-likelihood filtering for speckle reduction of ultrasound images [J]. IEEE Transactions on Medical Imaging, 200726 (5) 712-727. 
[4] Gongdong Wang, Jie Xu, Zhenkuan Pan, Zhaojing Diao. Ultrasound image denoising using backward diffusion and framelet regularization [J]. Biomedical Signal Processing and Control, 2014 13 212-217.

[5] A. Buades, B. Coll, J.M. Morel. A review of image denoising algorithm, with a new one [J]. Multiscale Modeling \& Simulation, 20054 (2) 490-530.

[6] Shuai Li, Ximei Zhao, Guodong Wang. Nonlocal TV model for multiplicative noise with Rayleigh distribution removal [J]. Chinese Jounal of Scientific Instrument, 201536 (7) 1570-1576.

[7] Michael Elad, Michal Aharon. Image denoising via sparse and redundant representations over learned dictionaries [J]. IEEE Transactions on Image Processing, 200615 (12) 3736-3745.

[8] Yong-Qiang Zhao, Jingxiang Yang. Hyperspectral image denoising via sparse representation and low-rank constraint [J]. IEEE Tansactions on Geoscience Remote Sensing, 2015 53(1) 296-308.

[9] Bhabesh Deka, Prabin Bora. Removal of correlated speckle noise using sparse and overcomplete representations [J]. Biomedical Signal Processing and Control, 20138 (6) 520-533.

[10]Tom Goldstein, Stanley Osher. The split Bregman method for l1-regularized problems [J]. SIAM Journal on Imaging Sciences, 20092 (2) 323-343.

[11]Fengtao Xiang, Zhengzhi Wang. Split Bregman iteration solution for sparse optimization in image restoration [J]. Optik, 2014125 5635-5640.

[12]YC Pati, R Rezaiifar, PS Krishnaprasad. Orthogonal matching pursuit: Recursive function approximation with applications to wavelet decomposition, Proc. 27th Asilomar Conf. Signals Syst. Comput. [C].New York: IEEE, 1993 629-639.

[13]L. Vese, S. Osher. Modeling textures with total variation minimization and oscillating patterns in image processing [J]. J. Sci.Comput., 200319 (11) 553-572.

[14]Zhou Wang, A.C. Bovik, H.R. Sheikh, et al.. Image quality assessment: from error visibility to structural similarity [J]. IEEE Transactions on Image Processing, 200413 (4) 600-612. 